Ergod. Th. \& Dynam. Sys. (First published online 2005), 0, 1-20*

doi:10.1017/S0143385704001038 C 2005 Cambridge University Press

* Provisional-final page numbers to be inserted when paper edition is published

\title{
Algebraic proof of the non-integrability of Hill's problem
}

\author{
JUAN J. MORALES-RUIZ $\dagger$, CARLES SIMÓ $\ddagger$ and SERGI SIMON $\ddagger$ \\ $\dagger$ Departament de Matemàtica Aplicada II, Universitat Politècnica de Catalunya, \\ Pau Gargallo, 5, 08028 Barcelona, Spain \\ (e-mail: juan.morales-ruiz@upc.es) \\ † Departament de Matemàtica Aplicada i Anàlisi, Universitat de Barcelona, \\ Gran Via, 585, 08007 Barcelona, Spain \\ (e-mail:carles@maia.ub.es,sergi@mat.ub.es)
}

(Received 22 September 2004 and accepted in revised form 11 November 2004)

Abstract. Hill's lunar problem appears in celestial mechanics as a limit of the restricted three-body problem. It is parameter-free and thus globally far from any simple well-known problem, and has shed strong numerical evidence of its lack of integrability in the past. An algebraic proof of meromorphic non-integrability is presented here. Beyond the result itself, the paper can also be considered as an example of the application of differential Galois and Morales-Ramis theories to a significant problem.

\section{Introduction}

Hill's problem (HP), usually dubbed lunar as a homage to its earliest motivation, or planar in order to distinguish it from its own extension to $\mathbb{R}^{3}$, is a model originally based on the Moon's motion under the joint influence of Earth and Sun [4]. A first simplification of the general three-body problem consists in assuming the Moon's mass is negligible and the primaries (Earth and Sun) move in circular orbits around their common barycentre. We then have a Hamiltonian system called the restricted three-body problem (RTBP, see [16]). Let $\mu=M_{E} /\left(M_{E}+M_{S}\right)$ and use a rotating coordinate frame whose first axis is spanned by the primaries. By a suitable choice of mass, length and time units we obtain the best-known equations for the RTBP:

$$
\left.\begin{array}{l}
\xi^{\prime \prime}-2 \eta^{\prime}=\Omega_{\xi}, \\
\eta^{\prime \prime}+2 \xi^{\prime}=\Omega_{\eta},
\end{array}\right\} \quad \Omega(\xi, \eta)=\frac{1-\mu}{r_{1}}+\frac{\mu}{r_{2}}+\frac{1}{2}\left(\xi^{2}+\eta^{2}\right), \quad\left\{\begin{array}{l}
r_{1}^{2}=(\xi-\mu)^{2}+\eta^{2}, \\
r_{2}^{2}=(\xi-\mu+1)^{2}+\eta^{2},
\end{array}\right.
$$

that is, $\Omega$ is the gravitational plus the centrifugal potential. Setting the Earth as the origin of coordinates and scaling length by $\mu^{1 / 3}$, HP is now defined by taking $\mu \rightarrow 0$ in the resulting equations. Thus, the RTBP can be written as an $O\left(\mu^{1 / 3}\right)$ perturbation of HP 
in a neighbourhood of the Earth of size $O\left(\mu^{1 / 3}\right)$ in the initial variables of the RTBP. HP itself does not depend on any parameter other than the energy and is therefore far enough, globally, from any known integrable system. The simplest expression known to date amounts to the polynomial of degree 6 shown by (6) in $\S 3$.

HP displays most of the numerical evidence inherent to chaotic dynamical systems. Hence, establishing its non-integrability in a rigorous way has long been a tempting, if elusive, goal; for instance, monodromy groups for the normal variational equations apparently invariably yield resonant matrices, thus discarding the application of Ziglin's theorem (see [18] for details). Our ultimate approach has been the use of the MoralesRamis theorem which connects two notions: integrability of Hamiltonian systems and integrability of linear systems of equations. Thanks to this main result, a symplectic change and a series of minor operations, we have afforded the proof avoiding burdensome calculations and strict dependence on numerical results.

Section 2 introduces the basic theory needed. Section 3 exposes the actual problem and states the main results. The next three sections are the main body of the proof. Its first part (corresponding to §4) is based on the computation of a particular solution of the HP; this solution and the sort of integral curve $\Gamma$ it determines, are in turn useful for the second part, described in $\$ 5$ and consisting of the layout (and a fundamental matrix $\Psi$ ) of the variational equations of the HP along $\Gamma$. The information we need about the matrix, included in $\$ 5.3$, is actually less than computing the whole of $\Psi$ explicitly, as we will see in §6: the study of the Galois differential group of the Picard-Vessiot extension for the aforementioned variational equations. This will be the concluding part of our proof, using the relevant facts concerning $\Psi$ to apply the Morales-Ramis theorem. All through the whole process, we shall make no more forays than necessary into the topics of special functions, representation theory and algebraic geometry.

Concerning the recent papers $[7,11]$ devoted to the very same goal through different techniques, in $\$ 7$ we expand on a comment regarding their authors' hypotheses.

As for most of the notation, real and complex vectors will be denoted in boldface and their Euclidean norms will be written in ordinary face. There will only be one independent variable $t$ properly regarded as time, and thus ${ }^{\prime}=d / d t$ all through the text. Given a field $K$ and $n \in \mathbb{N}, \mathcal{M}_{n}(K)$ is the ring of all square $n \times n$ matrices with their entries in $K$ and the group of regular matrices of $\mathcal{M}_{n}(K)$ is written $\mathrm{GL}_{n}(K)$ as usual. The field of all meromorphic functions defined on a Riemann surface $\Gamma$ is denoted $\mathcal{M}(\Gamma)$.

\section{Two notions of integrability}

The idea underlying any apprehension of an integrable dynamical system is the ability to make global assertions of the system's evolution with respect to time. The outcome of such assertions is sometimes called a solution, although giving them a strict definition has always proved a troublesome task, since each field of study has its own specialized notion of 'solvability', seldom equivalent to the others'. The two distinct notions described here do have a common trait: the ability to perform integration by quadratures, that is, resolution through 'algebraic' operations (including the inversion of functions) and 'quadratures', that is, the computation of integrals of known functions. 
2.1. Integrability of Hamiltonian systems. Let us restrict our attention to the case in which the system is Hamiltonian:

$$
q_{i}^{\prime}=\frac{\partial H}{\partial p_{i}}, \quad p_{i}^{\prime}=-\frac{\partial H}{\partial q_{i}}, \quad i=1, \ldots, n ;
$$

further details may be found in $[\mathbf{1 , 8}, \mathbf{1 5}]$. The following result does not merely provide for some Hamiltonians a description of their phase spaces; it also confers on the whole area a precise notion of integrability.

THEOREM 2.1. (Liouville-Arnold) Let $X_{H}$ be an n-degree-of-freedom real Hamiltonian having $n$ functionally independent first integrals $f_{1}=H, f_{2}, \ldots, f_{n}$ in pairwise involution. Let $\boldsymbol{a} \in \mathbb{R}^{n}$ and $M(\boldsymbol{a})=\left\{f_{i}(z)=a_{i}, i=1, \ldots, n\right\}$ be a non-critical-level manifold of $f_{1}, \ldots, f_{n}$. Then,

(1) $\quad M(\boldsymbol{a})$ is an invariant manifold of $X_{H}$;

(2) if compact and connected, $M(\boldsymbol{a})$ is diffeomorphic to $\mathbb{T}^{n}=\mathbb{R}^{n} / \mathbb{Z}^{n}$, and in a neighbourhood of the former there exists a coordinate system $(\boldsymbol{I}, \boldsymbol{\phi}) \in \mathbb{R}^{n} \times \mathbb{T}^{n}$ in which (1) reads

$$
I_{i}^{\prime}=0, \quad \phi_{i}^{\prime}=\omega_{i}, \quad i=1, \ldots, n,
$$

with $\omega_{i}=\omega_{i}(\boldsymbol{I}), i=1, \ldots, n$. In particular, $X_{H}$ can be integrated by quadratures.

For a proof, see [1]. Directly after the above theorem, we call an $n$-degree-of-freedom Hamiltonian $H$ integrable in the sense of Liouville-Arnold, completely integrable or simply integrable (and extend this definition to $X_{H}$ and (1)) if it has $n$ functionally independent integrals $f_{1}=H, f_{2}, \ldots, f_{n}$ in involution.

Remark 2.1. Although we have restricted everything to real variables, the Hamiltonian formulation may also be defined in the complex setting, thus allowing time and the canonical variables to be complex and the functions and fields to be analytical or meromorphic. The only nuisance for some purposes, though, is the absence of a complex analogue to Theorem 2.1 except for special cases (see [8]).

2.2. Integrability of linear differential systems. In the context of linear differential equations, the concept of integrability is conventionally limited to the possibility of solving the equation in terms of algebraic functions, integrals and exponentials of known functions or any finite combination of all three. This second notion is naturally inscribed in differential Galois theory as will be seen in Definition 2.6 and Theorem 2.7. Every single fact stated here is described in detail in reference [14].

Let $K$ be a field. A derivation on $K$ is an additive map $\partial: K \rightarrow K$ satisfying

$$
\partial(a b)=\partial(a) b+a \partial(b), \quad a, b \in K \quad \text { (Leibnitz rule). }
$$

A differential field is a pair $\left(K, \partial_{K}\right)$ consisting of a field and a derivation on it. The constants are the elements of the subfield $\operatorname{Const}(K):=\operatorname{ker} \partial_{K}$ of $K$. We henceforth denote $\partial=\partial_{K}$ unless necessary, and write $a^{\prime}$ (respectively, $a^{(n)}$ ) instead of $\partial(a)$ (respectively $\partial^{n}(a)$ ); we will also use this notation for elements of $K^{n}$, extending the derivation entrywise. 
An extension of differential fields, usually denoted $L \mid K$, is an inclusion $L \supset K$ such that $\left.\partial_{L}\right|_{K} \equiv \partial_{K}$. All fields and extensions will be assumed to be differential from this point on. We assume characteristic zero for every field considered. The set of all $K$-automorphisms of any differential extension $L \mid K$ (i.e. field isomorphisms $\sigma: L \rightarrow L$ such that $\left.\sigma\right|_{K} \equiv \operatorname{Id}_{K}$ and $\partial \circ \sigma \equiv \sigma \circ \partial$ ) is a group under map composition and will be denoted by $\operatorname{Aut}_{K}(L)$. Given any $m \in \mathbb{N}$, and using the propagation of morphism axioms of any $\sigma \in \operatorname{Aut}_{K}(L)$ to elements of $\mathcal{M}_{m}(K)$,

$$
\begin{aligned}
\left(\sigma\left(a_{i, j}\right)\right)_{1 \leq i, j \leq m}\left(\sigma\left(b_{i, j}\right)\right)_{1 \leq i, j \leq m} & =\left(\sum_{r=1}^{m} \sigma\left(a_{i, r}\right) \sigma\left(b_{r, j}\right)\right)_{1 \leq i, j \leq m} \\
& =\left(\sigma\left(\sum_{r=1}^{m} a_{i, r} b_{r, j}\right)\right)_{1 \leq i, j \leq m},
\end{aligned}
$$

we will indulge in as many abuses of notation as necessary when extending $\sigma$ entrywise to any $m \times m$ matrix.

Given a linear homogeneous differential system

$$
\boldsymbol{y}^{\prime}=A \boldsymbol{y}, \quad A \in \mathcal{M}_{n}(K)
$$

and an extension $E \mid K$ containing a set $V$ of solutions of (2), there is always a minimal subfield $L \subset E$ containing both $K$ and the entries of the elements of $V$; we write $L=K(V)$ and say $L$ is generated over $K$ as a differential field by the entries of elements of $V$. Since (2) is linear and homogeneous, $V$ is a Const $(L)$-vector space of dimension at most $n$. $\operatorname{Aut}_{K}(L)$ preserves $V$ and acts on it as a group of linear transformations over $\operatorname{Const}(K)$, and if $\operatorname{Const}(L)=\operatorname{Const}(K)$ the restriction of $\operatorname{Aut}_{K}(L)$ to $V$ gives a faithful representation $\operatorname{Aut}_{K}(L) \rightarrow \mathrm{GL}(V)$. $V$ owes its relevance to those situations in which it is precisely defined as the maximal set of linearly independent solutions of (2), thus begetting the differential analogue of a Galois extension; such an analogue corresponds to the case $\operatorname{dim}_{\text {Const }(L)}(V)=n$ and actually matches the situation in which no new constants are added to $K$.

Definition 2.2. $L \mid K$ is a Picard-Vessiot $(P-V)$ extension of for (2) if:

(1) $\operatorname{Const}(L)=\operatorname{Const}(K)$;

(2) there exists a fundamental matrix $\Phi \in \mathrm{GL}_{n}(L)$ for the equation; and

(3) $L$ is generated over $K$ as a differential field by the entries of $\Phi$.

Given a $P-V$ extension $L \mid K$ for (2) and an intermediate extension $L \supset L_{1} \supset K$ then $L \mid L_{1}$ is also a $P-V$ extension for some linear O.D.E. over $L_{1}$. We are calling $L \mid K$ a Picard-Vessiot extension if it is $P-V$ for some linear O.D.E. over $K$; an intrinsic definition may indeed be made, regardless of the equation. For the sake of simplicity and concreteness we are henceforth assuming all fields considered have $\mathbb{C}$ as field of constants. This assumption also assures existence and uniqueness of $P-V$ extensions.

An essential property of $P-V$ extensions is normality: for any $a \in L \backslash K$, there is a differential $K$-automorphism $\sigma$ of $L$ such that $\sigma(a) \neq a$.

Definition 2.3. If $L \mid K$ is a Picard-Vessiot extension for (2), then $\operatorname{Aut}_{K}(L)$ will be denoted $\operatorname{Gal}(L \mid K)$ and called the Galois differential group of $L$ over $K$ (or of the equation). 
Definition 2.4. A subgroup of $\mathrm{GL}_{n}(\mathbb{C})$, for some $n \in \mathbb{N}$, is a linear algebraic group provided its elements are matrices whose coefficients are zeros of polynomials in $\mathbb{C}$. A subgroup of a linear algebraic group is closed if it is itself a linear algebraic group.

The Galois differential group of equation (2) is a linear algebraic group; indeed, given a fundamental matrix $\Phi \in M_{n}(L), \sigma(\Phi)$ is also a fundamental matrix and hence $\sigma(\Phi)=$ $\Phi R(\sigma)$ with $R(\sigma) \in \mathrm{GL}_{n}(\mathbb{C})$, which yields an $n$-dimensional faithful representation

$$
\rho: \operatorname{Gal}(L \mid K) \rightarrow \mathrm{GL}_{n}(\mathbb{C}), \quad \sigma \mapsto R(\sigma)
$$

this renders $\operatorname{Gal}(L \mid K)$ a linear group. For a proof of its being also closed, see [14, Theorem 1.27]. Furthermore, the monodromy group of an equation (2), attained through analytical continuation of solutions, is a (not necessarily closed) subgroup of the differential Galois group of the corresponding $P-V$ extension. Whenever $G$ is the differential Galois group of some $P-V$ extension, we are identifying elements $\sigma$ of $G$ with the corresponding matrices $R(\sigma)$ defining representation $\rho$ in (3). In other words, we will be dealing either with the linear algebraic group $G$ or the matrix group $\rho(G)$.

We now state the so-called fundamental theorem of differential Galois theory.

THEOREM 2.5. Let $L \mid K$ be a Picard-Vessiot extension with common field of constants $\mathbb{C}$. Furthermore, let $G=\operatorname{Gal}(L \mid K), \mathcal{S}$ be the set of closed subgroups of $G$ and $\mathcal{L}$ the set of differential subfields of L. Define $\alpha: \mathcal{S} \rightarrow \mathcal{L}, \alpha(H)=L^{H}$, i.e. the subfield of $L$ formed by $H$-invariant elements; let $\beta: \mathcal{L} \rightarrow \mathcal{S}, \beta\left(L_{1}\right)=\operatorname{Gal}\left(L \mid L_{1}\right)$, the subgroup of $G$ of $L_{1}$-linear differential automorphisms of $L$. Then:

(1) $\alpha$ i $\beta$ are mutual inverses;

(2) the following are equivalent:

(a) $H \in \mathcal{S}$ is a normal subgroup of $G$;

(b) $L_{1}:=\alpha(H)=L^{H}$ is a $P-V$ extension of $K$;

and in such case $\operatorname{Gal}\left(L \mid L_{1}\right)=H$ and $\operatorname{Gal}\left(L_{1} \mid K\right) \simeq G / H$.

As foretold at the start of this subsection, we are now introducing the strict definition of what is to be called an integrable linear differential equation; it is precisely one whose $P-V$ extension falls into the following category.

Definition 2.6. Let $K$ be a differential field. $L \mid K$ is called a Liouville extension if no new constants are added and there exists a tower of extensions

$$
K=L_{0} \subset L_{1} \subset \cdots \subset L_{n}=L
$$

such that for $i=1, \ldots, n, L_{i}=L_{i-1}\left(t_{i}\right)$ and one of the following holds:

(1) $t_{i}^{\prime} \in L_{i-1}$; we say $t_{i}$ is an integral (of an element of $L_{i-1}$ );

(2) $t_{i} \neq 0$ and $t_{i}^{\prime} / t_{i} \in L_{i-1}$; in such case, $t_{i}$ is an exponential (of an integral of an element of $L_{i-1}$ );

(3) $t_{i}$ is algebraic over $L_{i-1}$.

If $L$ is a Liouville extension of $K$ and all $t_{i}$ are integrals (respectively exponentials), we say $L$ is an extension by integrals (respectively exponentials) of $K$.

What comes next, finally, is the fundamental characterization of Liouville extensions. 
THEOREM 2.7. Let $L$ be a Picard-Vessiot extension of $K$ with Galois differential group $G$. Then, the following are equivalent:

(1) $L$ is a Liouville extension of $K$;

(2) the identity component $G^{0}$ of $G$ is solvable.

Remark 2.2. Regarding $P-V$ extensions defined by integrals:

(1) Any quadrature $\int f$ of an element $f \in K$ is either again in $K$ or transcendental (i.e. solution to no polynomial equation with its coefficients in $K)$. Thus, $K\left(\int f\right)$ is either trivial or transcendental.

(2) If a Picard-Vessiot extension is defined only by quadrature adjunction,

$$
L=K\left(\int f_{1}, \int f_{2}, \ldots, \int f_{k}\right),
$$

where $f_{1}, f_{2}, \ldots, f_{k} \in K$, its Galois group is equal to $\left(\mathbb{C}_{+}\right)^{s}, s \leq k$. Here $\mathbb{C}_{+}$denotes the additive group of $\mathbb{C}$. Indeed, $\operatorname{Gal}(L \mid K)$ acts on quadratures in an additive manner and the only algebraic subgroups of $\mathbb{C}_{+}$are itself and the trivial group. See, for instance, $[\mathbf{1 4}$, Exercise 1.35 (1)].

2.3. The Morales-Ramis theorem. At this point, we need to rely on $\S \S 2.1$ and 2.2 despite having an initially real Hamiltonian. This is trivial for $\$ 2.2$ but is definitely not so for \$2.1, as stated in Remark 2.1; the usual procedure is to work on complex Hamiltonian systems (whether holomorphic or meromorphic) such that if the coordinates are real then Hamilton's equations are also real, i.e. systems which, restricting time and canonical variables to real values, reduce to the real system. We may then apply the Liouville-Arnold theorem to the real system and then consider all variables (dependent and independent) as complex. Fortunately, this is overall the case for HP.

As mentioned in $\S 1$, the theorem bearing this subsection's name connects the two notions of solvability listed in $\$ 2.1$ and $\$ 2.2$, namely as applied to a Hamiltonian $X_{H}$ and the linear variational equations $(\mathrm{VE}), \boldsymbol{\xi}^{\prime}=X_{H}^{\prime}(\widehat{z}(t)) \xi$ along an integral curve $\Gamma:=$ $\{\widehat{z}(t): t \in I\}$ of $X_{H}$, respectively. Actually, the theorem is the heuristic implementation of the following idea: if a Hamiltonian is integrable, then its variational equations must also be integrable.

The base field for the $P-V$ extension (i.e. the one containing the coefficients of the variational equations) is the field $\mathcal{M}(\Gamma)$ of meromorphic functions defined on the integral curve of $X_{H}$.

THEOREM 2.8. (Morales-Ramis) Assume there exist $n$ independent meromorphic first integrals in involution for $X_{H}$ in a neighbourhood of an integral curve $\Gamma$. Then, the identity component $G^{0}$ of the Galois group of the variational equations along $\Gamma$ is commutative.

Proof. See [9, Corollary 8] (or [8, Theorem 4.1]).

Remark 2.3. Reference [10] extends this result to higher order variationals; for an exposition of its conjecture phase, see [8, Section 8.3]. 
3. Setting and main results

3.1. Hill's problem. Let us write the simplest existing Hamiltonian formulation of HP. Everything listed here is described in detail in [12] and, especially, [13].

After following the steps listed in the introduction (including the limit-taking in $\mu$ ), we obtain

$$
\left.\begin{array}{l}
\bar{q}_{1}^{\prime \prime}=-\frac{\bar{q}_{1}}{\left(\bar{q}_{1}^{2}+\bar{q}_{2}^{2}\right)^{3 / 2}}+2 \bar{q}_{2}^{\prime}+3 \bar{q}_{1} \\
\bar{q}_{2}^{\prime \prime}=-\frac{\bar{q}_{2}}{\left(\bar{q}_{1}^{2}+\bar{q}_{2}^{2}\right)^{3 / 2}}-2 \bar{q}_{1}^{\prime}
\end{array}\right\}
$$

which are the best-known equations of HP. The HP Hamiltonian for the above equations (4) is

$$
H_{H P}(\overline{\boldsymbol{q}}, \overline{\boldsymbol{p}}):=\bar{p}^{2}-\bar{q}^{-1}+\left(\bar{p}_{1} \bar{q}_{2}-\bar{p}_{2} \bar{q}_{1}\right)+\frac{1}{2}\left(\bar{q}_{2}^{2}-2 \bar{q}_{1}^{2}\right) .
$$

The next steps are Levi-Civita regularization, a formulation of the problem in the extended phase space, a generalized canonical transformation and a scaling. The final expression is

$$
\mathcal{H}(\boldsymbol{Q}, \boldsymbol{P})=\mathrm{H}_{2}+\mathrm{H}_{4}+\mathrm{H}_{6},
$$

a sum of homogeneous polynomials of degrees 2,4 and 6 , respectively:

$H_{2}=P^{2} / 2+Q^{2} / 2, \quad H_{4}=-2 Q^{2}\left(P_{2} Q_{1}-P_{1} Q_{2}\right), \quad H_{6}=-4 Q^{2}\left(Q_{1}^{4}-4 Q_{1}^{2} Q_{2}^{2}+Q_{2}^{4}\right)$.

Our main statements and proofs (that is, the rest of $\S 3$ and $\S \S 4-6)$ will stem from Hamiltonian (6).

3.2. Statement of the main results. A first lemma will restrict our study to a particular solution of HP contained in an affine submanifold of the phase space $\mathbb{A}_{\mathbb{C}}^{4}$; we call it an invariant plane solution.

LEMMA 3.1. $X_{\mathcal{H}}$ has a particular solution (depending on the energy level $h$ ) of the form

$$
\left(Q_{1}(t), Q_{2}(t), P_{1}(t), P_{2}(t)\right)=\frac{1}{\sqrt{2}}\left(\phi(t), i \phi(t), \phi^{\prime}(t), i \phi^{\prime}(t)\right) .
$$

For all $0<h<1 /(6 \sqrt{3}), \phi^{2}(t)$ is elliptic with two simple poles in each period parallelogram.

Using this and properties of the specific elliptic function involved in $\phi(t)$, we then obtain the following.

LEMMA 3.2. The variational equations $(V E)$ of $X_{\mathcal{H}}$ along solution (7) have a fundamental matrix of the form

$$
\Psi(t)=\left(\begin{array}{cc}
\Phi_{N}(t) & \Phi_{N}(t) \\
0 & \int_{0}^{t} V(\tau) d \tau \\
0 & \Phi_{N}(t)
\end{array}\right),
$$

where

$$
\Phi_{N}(t)=\left(\begin{array}{cc}
\xi_{1}(t) & \xi_{2}(t) \\
i \xi_{1}^{\prime}(t) & i \xi_{2}^{\prime}(t)
\end{array}\right)
$$


is a fundamental matrix of the normal variational equations; furthermore, $\xi_{2}$ is a linear combination of elliptic functions and non-trivial elliptic integrals of first and second classes, and $\int_{0}^{t} V(\tau) d \tau$ is a $2 \times 2$ matrix function containing logarithmic terms in its diagonal.

This allows a careful study of the $P-V$ extension for the (VE) yielding the following.

THEOREM 3.3. The identity component $G^{0}$ of the Galois differential group of (VE) is noncommutative.

This proved, Theorem 2.8 gives the main result.

COROLlary 3.4. Hill's problem does not admit a meromorphic integral of motion independent of its Hamiltonian.

\section{Proof of Lemma 3.1}

4.1. Change of variables. Matrix

$$
A=\frac{1}{\sqrt{2}}\left(\begin{array}{ll}
1 & i \\
i & 1
\end{array}\right)
$$

provides for a symplectic change of variables,

$$
\left(\begin{array}{l}
Q_{1} \\
Q_{2}
\end{array}\right)=A\left(\begin{array}{l}
\bar{Q}_{1} \\
\bar{Q}_{2}
\end{array}\right), \quad\left(\begin{array}{l}
P_{1} \\
P_{2}
\end{array}\right)=\left(A^{-1}\right)^{T}\left(\begin{array}{l}
\bar{P}_{1} \\
\bar{P}_{2}
\end{array}\right),
$$

which in turn transforms Hamiltonian (6) into

$$
\overline{\mathcal{H}}=i\left(\bar{Q}_{1} \bar{Q}_{2}-\bar{P}_{1} \bar{P}_{2}\right)-4 i\left(3 \bar{Q}_{1}^{4}-2 \bar{Q}_{1}^{2} \bar{Q}_{2}^{2}+3 \bar{Q}_{2}^{4}\right) \bar{Q}_{1} \bar{Q}_{2}-4 \bar{Q}_{1} \bar{Q}_{2}\left(\bar{Q}_{1} \bar{P}_{1}-\bar{Q}_{2} \bar{P}_{2}\right) .
$$

The corresponding differential system $\bar{z}^{\prime}=X_{\overline{\mathcal{H}}}(\bar{z})$ now displays two invariant planes

$$
\pi_{1}:\left\{\bar{Q}_{2}=\bar{P}_{1}=0\right\}, \quad \pi_{2}:\left\{\bar{Q}_{1}=\bar{P}_{2}=0\right\},
$$

in any of which all non-trivial information of that system reduces to a hyperelliptic equation,

$$
\phi^{\prime \prime}=-\phi+12 \phi^{5}
$$

which through multiplication by $\phi^{\prime}$ and subsequent integration becomes

$$
\left(\phi^{\prime}\right)^{2}=-\phi^{2}+4 \phi^{6}+2 h .
$$

Defining $w=\phi^{2}, z=2 \phi \phi^{\prime}$, we arrive at the system

$$
w^{\prime}=z, \quad z^{\prime}=4\left(-w+8 w^{3}+h\right),
$$

whose Hamiltonian (at level-zero energy) is $\mathcal{K}(w, z)=\frac{1}{2} z^{2}+2 w^{2}-8 w^{4}-4 h w$.

Remark 4.1. The fact that in these invariant planes everything becomes simpler has a clear mechanical meaning. Some difficulties appear in (6) due to the presence of $H_{4}$, which mixes positions and momenta. It corresponds to the Coriolis term coming from the rotating frame. The present choice of variables singles out (complex) planes in which this term becomes zero. 
4.2. Solution of the new equation. The solution to system (10), or equivalently to equation $\left(w^{\prime}\right)^{2}=-4 w^{2}+16 w^{4}+8 h w$, is the inverse of an elliptic integral:

$$
t= \pm \int_{0}^{w(t)}\left(-4 y^{2}+16 y^{4}+8 h y\right)^{-1 / 2} d y+K_{1}, \quad K_{1} \in \mathbb{C},
$$

translation $t \mapsto t-K_{1}$ being the next obvious step. It is a known fact (see [17]) that given a polynomial of degree 4 without repeated factors, $p_{4}(x)=a_{4} x^{4}+4 a_{3} x^{3}+6 a_{2} x^{2}+4 a_{1} x+$ $a_{0}$, and defining constants (called invariants)

$$
g_{2}=a_{4} a_{0}-4 a_{3} a_{1}+3 a_{2}^{2}, \quad g_{3}=a_{0} a_{2} a_{4}+2 a_{1} a_{2} a_{3}-a_{2}^{3}-a_{4} a_{1}^{2}-a_{3}^{2} a_{0},
$$

then the solution for $t=\int_{a}^{w(t)}\left(p_{4}(x)\right)^{-1 / 2} d x$ is the following:

$$
w(t)=a+\frac{\sqrt{p_{4}(a)} \wp^{\prime}\left(t ; g_{2}, g_{3}\right)+\frac{1}{2} p_{4}^{\prime}(a)\left[\wp\left(t ; g_{2}, g_{3}\right)-\frac{1}{24} p_{4}^{\prime \prime}(a)\right]+\frac{1}{24} p_{4}(a) p_{4}^{\prime \prime \prime}(a)}{2\left[\wp\left(t ; g_{2}, g_{3}\right)-\frac{1}{24} p_{4}^{\prime \prime}(a)\right]^{2}-\frac{1}{48} p_{4}(a) p_{4}^{(4)}(a)},
$$

where $\wp\left(t ; g_{2}, g_{3}\right)$ is the Weierstrass elliptic function. In our specific case, this becomes

$$
w(t)=6 h / F(t), \quad z(t)=-18 h \wp^{\prime}\left(t ; g_{2}, g_{3}\right) / F^{2}(t),
$$

where $F(t):=3 \wp\left(t ; g_{2}, g_{3}\right)+1$. In particular,

$$
\phi_{1}(t)=\sqrt{(6 h / F(t))}, \quad \phi_{2}(t)=-\phi_{1}(t),
$$

are solutions to original equation (8). Furthermore, a simple calculation proves $h^{*}=$ $1 /(6 \sqrt{3})$ to be a separatrix value in which $\phi_{1}^{2}(t)=\phi_{2}^{2}(t)$ breaks down into combinations of hyperbolic functions. In order to step into the next subsection, we are therefore assuming $0<h<h^{*}$.

4.3. Singularities of $\phi^{2}(t)$. We are now proving that, for the above range of $h, w(t)$ has two simple poles in each period parallelogram, the sides of which will be denoted as $2 \omega_{1}, 2 \omega_{2}$, as usual. In virtue of [3, p. 96], expression $1 /\left(\wp(t)-\wp\left(t^{*}\right)\right)$ (in our case, $\left.\wp\left(t^{*}\right)=-1 / 3\right)$ has exactly two simple poles in $t^{*},-t^{*}\left(\bmod 2 \omega_{1}, 2 \omega_{2}\right)$, with respective residues $1 / \wp^{\prime}\left(t^{*}\right)$ and $-1 / \wp^{\prime}\left(t^{*}\right)$. Therefore, all double poles, if any, of $1 /\left(\wp(t)-\wp\left(t^{*}\right)\right)$, expanding around $t=t^{*}$, are precisely those $t^{*}$ such that $\wp^{\prime}\left(t^{*}\right)=0$. We have

$$
\left(\wp^{\prime}\left(t ; g_{2}, g_{3}\right)\right)^{2}=4\left(\wp\left(t ; g_{2}, g_{3}\right)\right)^{3}-g_{2} \wp\left(t ; g_{2}, g_{3}\right)-g_{3}=4 \wp^{3}-\frac{4}{3} \wp-\frac{8}{27}+64 h^{2},
$$

and every pole (whether double or not) must satisfy $\wp\left(t^{*}\right)=-1 / 3 ; X=-1 / 3$ is obviously not a root of $4 X^{3}-4 X / 3-8 / 27+64 h^{2}$ unless $h=0$. This ends the proof.

\section{Proof of Lemma 3.2}

5.1. Layout of the system. Reordering the vector of dependent canonical variables as $\left(\bar{Q}_{1}, \bar{P}_{2}, \bar{Q}_{2}, \bar{P}_{1}\right)^{T}$ and restricting ourselves to the particular solution found in $\S 4$,

$$
\bar{Q}_{1}=\phi, \quad \bar{Q}_{2}=0, \quad \bar{P}_{1}=0, \quad \bar{P}_{2}=i \phi^{\prime},
$$


the variational equations (VE) along that solution are written as

$$
\left(\begin{array}{l}
\bar{\xi}^{\prime} \\
\bar{\eta}^{\prime} \\
\xi^{\prime} \\
\eta^{\prime}
\end{array}\right)=\left(\begin{array}{cccc}
0 & -i & -4 w & 0 \\
i\left(60 w^{2}-1\right) & 0 & -4 i z & 4 w \\
0 & 0 & 0 & -i \\
0 & 0 & i\left(60 w^{2}-1\right) & 0
\end{array}\right)\left(\begin{array}{l}
\bar{\xi} \\
\bar{\eta} \\
\xi \\
\eta
\end{array}\right)=:\left(\begin{array}{cc}
A_{1} & B_{1} \\
0 & A_{1}
\end{array}\right)\left(\begin{array}{l}
\bar{\xi} \\
\bar{\eta} \\
\xi \\
\eta
\end{array}\right)
$$

and their lower right block, the normal variational equations (NVE)

$$
\left(\begin{array}{l}
\xi^{\prime} \\
\eta^{\prime}
\end{array}\right)=\left(\begin{array}{cr}
0 & -i \\
i\left(60 w^{2}-1\right) & 0
\end{array}\right)\left(\begin{array}{l}
\xi \\
\eta
\end{array}\right)
$$

that is,

$$
\xi^{\prime \prime}(t)=\left(60 w^{2}(t)-1\right) \xi(t) .
$$

The next step is to obtain a fundamental matrix for (12). An obvious short cut is to take $w$ as new independent variable and to define $\Xi(w), H(w)$ such that $\xi=\Xi \circ w$ and $\eta=H \circ w$. We have

$$
\frac{d^{2} \Xi}{d w^{2}}=4\left(\frac{w-8 w^{3}-h}{w f(w, h)}\right) \frac{d \Xi}{d w}+\frac{60 w^{2}-1}{w f(w, h)} \Xi,
$$

also expressible in matrix form

$$
\left(\begin{array}{l}
d / d w \Xi \\
d / d w H
\end{array}\right)=\frac{1}{\sqrt{w f(w, h)}}\left(\begin{array}{cr}
0 & -i \\
i\left(60 w^{2}-1\right) & 0
\end{array}\right)\left(\begin{array}{l}
\Xi \\
H
\end{array}\right),
$$

where $f=f(w, h)=4\left(4 w^{3}-w+2 h\right)$.

5.2. Fundamental matrix of the VE. We are now interested in the fundamental matrix of (11). Let us start from the block notation

$$
\Psi=\left(\begin{array}{ll}
P & Q \\
R & S
\end{array}\right),
$$

$P, Q, R, S$ being $2 \times 2$ matrices with their entries in some differential field to be described in $\S 6$. We can assume $\Psi(0)=\mathrm{Id}_{4}$, which, along with the triangular form of (11), assures $R \equiv 0$. In particular, the matrix form of the NVE (12) can be written as $S^{\prime}=A_{1} S$. Let us now proceed to integrate these normal equations. More precisely, let us make explicit all necessary information about the fundamental matrix $\Phi_{N}(t)$ of (12) with initial condition $\Phi_{N}(0)=\mathrm{Id}_{2}$.

Using well-known properties of $\wp^{\prime}$ and $\wp^{\prime \prime}$, it is easy to prove that $\Xi_{1}(w)=\sqrt{f(w, h)}$ is a solution of (14), and therefore $\xi_{1}(t)=\Xi_{1}(w(t))=\wp^{\prime}\left(t ; g_{2}, g_{3}\right)\left(3 \wp\left(t ; g_{2}, g_{3}\right)+\right.$ $1)^{-3 / 2}$ is a solution of (13). A first solution of (12) is then

$$
\left(\begin{array}{l}
\xi_{1}(t) \\
\eta_{1}(t)
\end{array}\right)=C_{1}\left(\begin{array}{c}
\left(16 w^{3}(t)-4 w(t)+8 h\right)^{1 / 2} \\
-2 i\left(12 w^{2}(t)-1\right) \sqrt{w(t)}
\end{array}\right), \quad C_{1} \in \mathbb{C} .
$$

We now recall d'Alembert's method [6, p. 122] in order to obtain a second solution of (13), independent of $\xi_{1}$. This solution is

$$
\xi_{2}(t)=\xi_{1}(t) \int_{0}^{t}\left\{\xi_{1}(\tau)\right\}^{-2} d \tau
$$


see $§ 5.3 .1$ for further details. After recovering our former independent variable $t$ through composition we have a fundamental matrix for the NVE, that is, the block $S$ in (16),

$$
\Phi_{N}(t)=\left(\begin{array}{ll}
\xi_{1} & \xi_{2} \\
\eta_{1} & \eta_{2}
\end{array}\right)=\left(\begin{array}{cc}
\xi_{1} & \xi_{2} \\
i \xi_{1}^{\prime} & i \xi_{2}^{\prime}
\end{array}\right) .
$$

In particular, $P(t) \equiv S(t)$ since they are both fundamental matrices for the same initial value problem. We now compute the block $Q$ in (16); the standing equations (in vector form) are

$$
\left(\begin{array}{l}
\bar{\xi}^{\prime} \\
\bar{\eta}^{\prime}
\end{array}\right)=\left(\begin{array}{cc}
0 & -i \\
i\left(60 w^{2}-1\right) & 0
\end{array}\right)\left(\begin{array}{l}
\bar{\xi} \\
\bar{\eta}
\end{array}\right)+\left(\begin{array}{cc}
-4 w & 0 \\
-4 i z & 4 w
\end{array}\right)\left(\begin{array}{l}
\xi \\
\eta
\end{array}\right)
$$

where $(\xi, \eta)^{T}$ are the solutions to the NVE. Applying variation of constants to (18) we obtain

$$
Q(t)=\Phi_{N}(t) \int_{0}^{t} V(\tau) d \tau
$$

where

$$
C(t)=\left(\begin{array}{cc}
-4 w(t) & 0 \\
-4 i z(t) & 4 w(t)
\end{array}\right), \quad V(t)=\Phi_{N}^{-1}(t) C(t) \Phi_{N}(t)
$$

In other words, the fundamental matrix of (11) has the form

$$
\Psi(t)=\left(\begin{array}{cc}
\Phi_{N}(t) & \Phi_{N}(t) \\
0 & \int_{0}^{t} V(\tau) d \tau \\
\Phi_{N}(t)
\end{array}\right) .
$$

Remark 5.1. In view of (19), computing $\Psi$ explicitly would now only take the computation of four integrals. The path we are taking, however, is a different one, although we are keeping in mind all of this notation and the final expression (20).

5.3. Relevant facts concerning $\Psi(t)$. As said in $\$ 1$ and in the above remark, we are not coping with the symbolic calculus needed to obtain (19) explicitly. Instead, our next aim is to prove only two specific properties of the fundamental matrix $\Psi$ of (11), namely the existence of first- and second-class elliptic integrals and logarithmic terms in its coefficients. The two consecutive steps of transcendence forced by these two new objects will provide the rest of our proof.

5.3.1. Elliptic integrals in $\Phi_{N}$. Let $K$ be the field of all elliptic functions of the complex plane. We know a solution of (13),

$$
\xi_{1}(t)=\left(4 w^{3}(t)-w(t)+2 h\right)^{1 / 2},
$$

and can obtain a second one using (17) and the chain rule. Let $\alpha_{1}, \alpha_{2}, \alpha_{3}$ be the values of $w$ for which $f(w, h)=0$, define the functions

$$
\beta(w, h):=\arcsin \left[\left(\frac{w\left(\alpha_{3}-\alpha_{1}\right)}{\alpha_{3}\left(w-\alpha_{1}\right)}\right)^{1 / 2}\right], \quad k(h):=\left(\frac{\alpha_{3}\left(\alpha_{1}-\alpha_{2}\right)}{\alpha_{2}\left(\alpha_{1}-\alpha_{3}\right)}\right)^{1 / 2},
$$

(both attaining complex, non-zero values if $h \in\left(0, h^{*}\right)$ and therefore $\left.w(t) \neq 0\right)$ and let

$$
E(\beta \mid k):=\int_{0}^{\beta}\left(1-k^{2} \sin ^{2} \theta\right)^{-1 / 2} d \theta, \quad F(\beta \mid k):=\int_{0}^{\beta}\left(1-k^{2} \sin ^{2} \theta\right)^{1 / 2} d \theta
$$


be the elliptic integrals of first and second class, respectively (see $[3,17])$. We then obtain a fundamental matrix for the NVE (15),

$$
\begin{aligned}
\bar{\Phi}_{N}(w) & =\left(\begin{array}{ll}
\Xi_{1}(w) & \Xi_{2}(w) \\
H_{1}(w) & H_{2}(w)
\end{array}\right) \\
& =\left(\begin{array}{cc}
\sqrt{f(w, h)} & g_{1}\left\{f_{1} E(\beta \mid k)+f_{2} F(\beta \mid k)+g_{2}\right\} \\
2 i \sqrt{w}\left(-1+12 w^{2}\right) & i(d / d w)\left(g_{1}\left\{f_{1} E(\beta \mid k)+f_{2} F(\beta \mid k)+g_{2}\right\}\right)
\end{array}\right),
\end{aligned}
$$

for some $f_{1}=f_{1}(h), f_{2}=f_{2}(h), g_{1}=g_{1}(w, h), g_{2}=g_{2}(w, h)$, the first three nonvanishing if $h \in\left(0, h^{*}\right)$, and the last two linked to $w$ by algebraic equations. In particular, this yields our fundamental matrix $\Phi_{N}(t)=\bar{\Phi}_{N}(w(t))$ for (12).

Remark 5.2. The fundamental trait of $E(\beta \mid k)$ and $F(\beta \mid k)$ is that they are transcendental over $K$. Indeed, non-trivial elliptic integrals of the first and second classes are not elliptic functions (see [3, Theorem 6.5 and its proof]) and they stem from quadratures; thus, as said in Remark 2.2(1), $E(\beta \mid k)$ and $F(\beta \mid k)$ cannot be expressed in terms of elliptic functions under any relation of algebraic dependence.

5.3.2. Logarithms in $\Psi$. Let us prove the existence of terms with non-zero residue in the diagonal of matrix $V(t)$. As

$$
\Phi_{N}(t)=\left(\begin{array}{ll}
\xi_{1} & \xi_{2} \\
\eta_{1} & \eta_{2}
\end{array}\right)=\left(\begin{array}{cc}
\xi_{1} & \xi_{2} \\
i \xi_{1}^{\prime} & i \xi_{2}^{\prime}
\end{array}\right)
$$

is the fundamental matrix of a Hamiltonian linear system, it is symplectic. The integrand in (19) becomes

$$
\begin{aligned}
V(t) & =4 i\left(\begin{array}{cc}
-w\left(\xi_{2} \xi_{1}^{\prime}+\xi_{1} \xi_{2}^{\prime}\right)+w^{\prime} \xi_{1} \xi_{2} & -\xi_{2}\left(2 \xi_{2}^{\prime} w-\xi_{2} w^{\prime}\right) \\
\left(2 w \xi_{1}^{\prime}-w^{\prime} \xi_{1}\right) \xi_{1} w\left(\xi_{1} \xi_{2}^{\prime}+\xi_{1}^{\prime} \xi_{2}\right)-w^{\prime} \xi_{1} \xi_{2} &
\end{array}\right) \\
& =: 4 i\left(\begin{array}{cr}
u(t) & v_{1}(t) \\
v_{2}(t) & -u(t)
\end{array}\right)
\end{aligned}
$$

For every $h \in\left(0, h^{*}\right)$, and taking profit of what was proved in $\S 4.3$, we expand these four entries around a simple pole $t^{*}$ of $w(t)$; expressing only the first term in each power series, we have

$$
\begin{gathered}
w(t)=C_{0}\left(t-t^{*}\right)^{-1}+O(1), \\
\xi_{1}(t)=2 C_{0}^{3 / 2}\left(t-t^{*}\right)^{-3 / 2}+O\left(\left(t-t^{*}\right)^{-1 / 2}\right), \\
\xi_{2}(t)=\frac{C_{0}^{-3 / 2}}{8}\left(t-t^{*}\right)^{5 / 2}+O\left(\left(t-t^{*}\right)^{7 / 2}\right),
\end{gathered}
$$

for some $C_{0}=C_{0}(h) \in \mathbb{C}$; therefore,

$$
\begin{gathered}
u(t)=-\frac{C_{0}}{2}\left(t-t^{*}\right)^{-1}+O(1), \\
v_{1}(t)=-\frac{3}{32 C_{0}^{2}}\left(t-t^{*}\right)^{3}+O\left(\left(t-t^{*}\right)^{4}\right), \\
v_{2}(t)=-8 C_{0}^{4}\left(t-t^{*}\right)^{-5}+O\left(\left(t-t^{*}\right)^{-4}\right) .
\end{gathered}
$$


Hence, and except for the only value of $h$ forcing $C_{0}=0$ (i.e. $h=0$ ), we have a non-zero residue in $u(t)$, which results in the aforementioned logarithmic terms in the diagonal of

$$
\int_{0}^{t} V(\tau) d \tau=\left(\begin{array}{cc}
\int_{0}^{t} u(\tau) d \tau & \int_{0}^{t} v_{1}(\tau) d \tau \\
\int_{0}^{t} v_{2}(\tau) d \tau & -\int_{0}^{t} u(\tau) d \tau
\end{array}\right) .
$$

Remark 5.3. As before, there appears a class of functions that cannot be linked algebraically to the former. Indeed, logarithms are special cases of elliptic integrals of the third class, which are neither elliptic functions nor elliptic integrals of first or second class (see [3, Theorem 6.5 and its proof] once more), and in this case the logarithms have been obtained through a quadrature. Remark 2.2(1) yields the rest.

We thus have a second transcendental extension of fields of functions; it is the combination of this with the previous extension that will ultimately render $G^{0}$ non-commutative.

\section{Proof of Theorem 3.3}

Let us interpret our results in terms of field extensions. First of all, we note that using coordinates $(x, y)=\left(\phi, \phi^{\prime}\right)$ all solutions of the equation (9) roam in the hyperelliptic curve

$$
\Gamma_{h}:=\left\{(x, y) \in \mathbb{C}^{2}: y^{2}=-x^{2}+4 x^{6}+2 h\right\} .
$$

Denote by $\mathrm{VE}_{\Gamma_{h}}$ the expression of $\operatorname{VE}(11)$ and $\widehat{G}:=\operatorname{Gal}\left(E V_{\Gamma_{h}}\right)$; let $\widehat{G}^{0}$ be the identity component of $\widehat{G}$. The previous transformation $w=x^{2}, z=2 x y$ induces a finite branched covering

$$
\Gamma_{h} \rightarrow \Lambda_{h},
$$

where $\Lambda_{h}$ is the elliptic curve defined by

$$
\Lambda_{h}:=\left\{z^{2} / 2+2 w^{2}-8 w^{4}-4 h w=0\right\}
$$

and the group $\widehat{G}^{0}$ does not change; this is a consequence of [9, Theorem 5] (see also [8, Theorem 2.5]), according to which the identity component of the Galois group remains invariant under covering maps of this sort. We may thus keep with the abuse in notation of calling $\widehat{G}$ and $\widehat{G}^{0}$ the Galois group of $\mathrm{VE}_{\Gamma_{h}}$ and its identity component, respectively, now in variable $w$.

Keeping $K\left(=\mathcal{M}\left(\Lambda_{h}\right)\right)$ as the field of all elliptic functions, let us make explicit the Picard-Vessiot extension over $K$ for $\mathrm{VE}_{\Gamma_{h}}$.

1. First of all, let us define the extension

$$
K \subset K_{1}:=K\left(\xi_{1}, \xi_{1}^{\prime}\right)
$$

based on the adjunction of the first solution $\xi_{1}$ of (12) and its derivative, which is an algebraic (in fact, quadratic) one. The identity component of the Galois group of this extension is, therefore, trivial. 
2. Second, adjoining to this new field the solution $\xi_{2}$ from (17) we obtain the extension

$$
K_{1} \subset L_{1}:=K_{1}\left(\xi_{2}, \xi_{2}^{\prime}\right)=K\left(\xi_{1}, \xi_{1}^{\prime}, \xi_{2}, \xi_{2}^{\prime}\right),
$$

which is transcendental, since it is non-trivial and defined exclusively by an adjunction of quadratures (see Remark 5.2).

3. Third, adjoining the matrix integral from (19) to $L_{1}$, we have

$$
L_{1} \subset L_{2}:=L_{1}\left(\int_{0}^{t} u, \int_{0}^{t} v_{1}, \int_{0}^{t} v_{2}\right),
$$

also given by quadratures, non-trivial, and thus transcendental, in virtue of Remark 5.3.

So far, the $P-V$ extension $L_{2} \mid K$ of the VE has been decomposed as a tower of $P-V$ extensions

$$
K \subset K_{1} \subset L_{1} \subset L_{2} .
$$

Let $\widehat{G}:=\operatorname{Gal}\left(L_{2} \mid K\right)$. The fact that each of above extensions results from adjoining either algebraic elements or quadratures renders $L_{2} \mid K$ a Liouville extension, and thus $\widehat{G}^{0}$ a solvable group. Our aim is to prove that the (stronger) condition demanded by Theorem 2.8 is not fulfilled, i.e. $\widehat{G}^{0}$ is not commutative. The proof of this fact has five steps:

Step 1. Since $L_{2} \mid K_{1}$ is transcendental and $K_{1} \mid K$ is algebraic, we may assume the base field of the tower to be $K_{1}$, for $\widehat{G}^{0} \cong \operatorname{Gal}\left(L_{2} \mid K_{1}\right)$; indeed, all of the contributions derived from transcendental elements stay in $\widehat{G}^{0}$, and the last part of Theorem 2.5 asserts

$$
\widehat{G} / \widehat{G}^{0} \cong \operatorname{Gal}\left(K_{1} \mid K\right) \text {. }
$$

This restricts our study to $\operatorname{Gal}\left(L_{2} \mid K_{1}\right)$, besides proving it connected and thus equal to its identity component; in a further abuse of notation, we may call it $\widehat{G}^{0}$ again.

Step 2. Let us prove that the elements $R(\sigma)$ of the Galois group $\widehat{G}^{0}=\operatorname{Gal}\left(L_{2} \mid K_{1}\right)$ are unipotent matrices of the following kind:

$$
\widehat{G}^{0}=\left\{\left(\begin{array}{cccc}
1 & \mu & A_{1} & A_{2} \\
0 & 1 & A_{3} & A_{4} \\
0 & 0 & 1 & \mu \\
0 & 0 & 0 & 1
\end{array}\right): \mu \in S_{0} ; A_{1}, A_{2}, A_{3}, A_{4} \in T_{0}\right\}
$$

for some subsets $S_{0}, T_{0} \subset \mathbb{C}$ such that $S_{0} \neq\{0\}$.

Indeed, writing $R(\sigma)$ in block notation, $R(\sigma)=\left(\begin{array}{ll}M_{1} & M_{2} \\ M_{3} & M_{4}\end{array}\right)$, equation $\sigma(\Psi)=\Psi R(\sigma)$ reads

$$
\begin{aligned}
\sigma(\Psi) & =\left(\begin{array}{cc}
\Phi_{N}(t) & \Phi_{N}(t) \int_{0}^{t} V(\tau) d \tau \\
0 & \Phi_{N}(t)
\end{array}\right)\left(\begin{array}{ll}
M_{1} & M_{2} \\
M_{3} & M_{4}
\end{array}\right) \\
& =\left(\begin{array}{cc}
\Phi_{N} M_{1}+\left(\Phi_{N} \int_{0}^{t} V\right) M_{3} & \Phi_{N} M_{2}+\left(\Phi_{N} \int_{0}^{t} V\right) M_{4} \\
\Phi_{N} M_{3} & \Phi_{N} M_{4}
\end{array}\right) \\
& =\left(\begin{array}{cc}
\sigma\left(\Phi_{N}\right) & \sigma\left(\Phi_{N} \int_{0}^{t} V\right) \\
\sigma(0) & \sigma\left(\Phi_{N}\right)
\end{array}\right) .
\end{aligned}
$$


From (22) and (23) we obtain $M_{1}=M_{4}$ and $M_{3}=0$. We are now working on $\operatorname{Gal}\left(L_{2} \mid K_{1}\right)$, and the first column of $\Phi_{N}(t)$ is $\left(\xi_{1}(t), i \xi^{\prime}(t)\right)^{T} \in K_{1}^{2}$; thus, $\sigma$ must leave it fixed. That is, defining

$$
M_{1}=M_{4}=\left(\begin{array}{ll}
a & b \\
c & d
\end{array}\right)
$$

we have

$$
\sigma\left(\begin{array}{cc}
\xi_{1} & \xi_{2} \\
i \xi_{1}^{\prime} & i \xi_{2}^{\prime}
\end{array}\right)=\left(\begin{array}{cc}
\xi_{1} & \xi_{1} b+\xi_{2} d \\
i \xi_{1}^{\prime} & i \xi_{1}^{\prime} b+i \xi_{2}^{\prime} d
\end{array}\right)
$$

so the first column in $M_{1}$ and $M_{4}$ must be $(a, c)^{T}=(1,0)^{T}$. Their second column must then be of the form $(\mu, 1)^{T}$ for some $\mu \in \mathbb{C}$, since $\sigma\left(\Phi_{N}(t)\right)=\Phi_{N}(t) M_{1}$ is symplectic. This altogether forces the given expression for the diagonal blocks in (21).

The actual domain of definition $S_{0}$ for $\mu$ will be seen in the next step, but we can already assert $\mu$ is not identically zero. If it were, then the action of $\widehat{G}^{0}$ would leave $\xi_{2}, \xi_{2}^{\prime} \in L_{2}$ fixed. This, the definition of $L_{2}, L_{1}$ and the normality of $P-V$ extensions would in turn imply $\xi_{2}, \xi_{2}^{\prime} \in K_{1}$, i.e. we would have elliptic integrals in an algebraic extension of the field of elliptic functions; as said in Remark 5.2, this is absurd. Consequently, $S_{0} \neq\{0\}$.

Step 3. Let us prove $S_{0}=\mathbb{C}$. Indeed, the action of $\widehat{G}^{0}$ on $\operatorname{diag}\left(\Phi_{\mathrm{N}}, \Phi_{\mathrm{N}}\right)$ is of the form

$$
\tilde{G}=\left\{\left(\begin{array}{cccc}
1 & \mu & 0 & 0 \\
0 & 1 & 0 & 0 \\
0 & 0 & 1 & \mu \\
0 & 0 & 0 & 1
\end{array}\right): \mu \in S_{0}\right\},
$$

itself a representation of the additive group $\mathbb{C}_{+}$, which in turn has only two algebraic subgroups, namely itself and $\{0\}$; step 2 already discarded the first case, so we are left with $S_{0}=\mathbb{C}$.

Step 4. We are now giving a new provisional form to our group. We already know $\sigma \Phi_{N}=\Phi_{N} M_{1}$; let us first study the action of any $\sigma \in \widehat{G}^{0}$ over the four entries of $\int V$. Applying the identity $\partial \circ \sigma \equiv \sigma \circ \partial$ on $\int V$ and integrating the resulting equation, we obtain

$$
\sigma \int \Phi_{N}^{-1} C \Phi_{N}=\int \sigma\left(\Phi_{N}^{-1} C \Phi_{N}\right)+M
$$

for some $M=\left(\begin{array}{ll}\delta & \gamma \\ \beta & \kappa\end{array}\right) \in \mathcal{M}_{2}(\mathbb{C})$. Besides, using $\sigma C=C$ we have

$$
\sigma\left(\Phi_{N}^{-1} C \Phi_{N}\right)=\left(\sigma \Phi_{N}\right)^{-1} C\left(\sigma \Phi_{N}\right)=M_{1}^{-1}\left(\Phi_{N}^{-1} C \Phi_{N}\right) M_{1},
$$

which translates (25) into

$$
\sigma \int \Phi_{N}^{-1} C \Phi_{N}=M_{1}^{-1}\left(\int \Phi_{N}^{-1} C \Phi_{N}\right) M_{1}+M
$$

that is, the following separate actions of $\sigma$ on the entries of $\int\left(\Phi_{N}^{-1} C \Phi_{N}\right)$ :

$$
\int\left(\begin{array}{c}
u \\
v_{1} \\
v_{2} \\
-u
\end{array}\right) \mapsto \int\left(\begin{array}{c}
u-\mu v_{2} \\
2 \mu u-\mu^{2} v_{2} \\
v_{2} \\
\mu v_{2}-u
\end{array}\right)+\left(\begin{array}{l}
\delta \\
\gamma \\
\beta \\
\kappa
\end{array}\right),
$$

the first and fourth components of which readily imply $\delta=-\kappa$. 
On the other hand, (26) allows us to write (22) in the equivalent form

$$
\sigma(\Psi)=\left(\begin{array}{cc}
\sigma \Phi_{N} & \left(\sigma \Phi_{N}\right)\left[M_{1}^{-1} M_{2}+\int \sigma\left(\Phi_{N}^{-1} C \Phi_{N}\right)\right] \\
0 & \sigma \Phi_{N}
\end{array}\right) .
$$

Morphism axioms (and (23)) render the latter's upper right block equal to $\left(\sigma \Phi_{N}\right)\left(\sigma \int \Phi_{N}^{-1} C \Phi_{N}\right)$, and thus force the following to hold,

$$
\sigma\left(\int \Phi_{N}^{-1} C \Phi_{N}\right)=M_{1}^{-1} M_{2}+\int \sigma\left(\Phi_{N}^{-1} C \Phi_{N}\right),
$$

which along with (27) yields $M_{1}^{-1} M_{2}=M$. This gives us the explicit form for the upper $2 \times 2$ block in the generic expression (21) for $R(\sigma)$ :

$$
M_{2}=M_{1} M=\left(\begin{array}{cc}
-\kappa+\mu \beta & \gamma+\mu \kappa \\
\beta & \kappa
\end{array}\right) .
$$

In particular,

$$
\widehat{G}^{0}=\left\{\left(\begin{array}{cccc}
1 & \mu & -\kappa+\mu \beta & \gamma+\mu \kappa \\
0 & 1 & \beta & \kappa \\
0 & 0 & 1 & \mu \\
0 & 0 & 0 & 1
\end{array}\right): \mu \in \mathbb{C}, \kappa \in S_{1}, \beta \in S_{2}, \gamma \in S_{3}\right\}
$$

for some subsets $S_{1}, S_{2}, S_{3} \subset \mathbb{C}$.

Step 5. Further specification of the domains of definition of $\kappa, \beta, \gamma$ will finish our proof. We already know $S_{0}=\mathbb{C}$ is the domain for $\mu$. Given any element $a_{\mu, \kappa, \beta, \gamma} \in \widehat{G}^{0}$, we have

$$
\begin{aligned}
a_{\mu, \kappa, \beta, \gamma} & =\left(\begin{array}{cccc}
1 & \mu & -\kappa+\mu \beta & \gamma+\mu \kappa \\
0 & 1 & \beta & \kappa \\
0 & 0 & 1 & \mu \\
0 & 0 & 0 & 1
\end{array}\right) \\
& =\left(\begin{array}{cccc}
1 & \mu & 0 & 0 \\
0 & 1 & 0 & 0 \\
0 & 0 & 1 & \mu \\
0 & 0 & 0 & 1
\end{array}\right)\left(\begin{array}{cccc}
1 & 0 & -\kappa & 0 \\
0 & 1 & 0 & \kappa \\
0 & 0 & 1 & 0 \\
0 & 0 & 0 & 1
\end{array}\right)\left(\begin{array}{llll}
1 & 0 & 0 & 0 \\
0 & 1 & \beta & 0 \\
0 & 0 & 1 & 0 \\
0 & 0 & 0 & 1
\end{array}\right)\left(\begin{array}{llll}
1 & 0 & 0 & \gamma \\
0 & 1 & 0 & 0 \\
0 & 0 & 1 & 0 \\
0 & 0 & 0 & 1
\end{array}\right) \\
& =U_{\mu} V_{\kappa} W_{\beta} X_{\gamma} .
\end{aligned}
$$

Assume, for the moment, $S_{1}=S_{2}=S_{3}=\mathbb{C}$. Defining $G$ and $H$ as the subgroups of $\widehat{G}^{0}$ generated by $U_{\mu}$ and $V_{\kappa} W_{\beta} X_{\gamma}$, respectively,

$$
G=\left\{\left(\begin{array}{llll}
1 & \mu & 0 & 0 \\
0 & 1 & 0 & 0 \\
0 & 0 & 1 & \mu \\
0 & 0 & 0 & 1
\end{array}\right): \mu \in \mathbb{C}\right\}, \quad H=\left\{\left(\begin{array}{cccc}
1 & 0 & -\kappa & \gamma \\
0 & 1 & \beta & \kappa \\
0 & 0 & 1 & 0 \\
0 & 0 & 0 & 1
\end{array}\right): \kappa, \beta, \gamma \in \mathbb{C}\right\},
$$

$G$ is a representation of $\mathbb{C}_{+}$, and $H$, unlike $G$, is a normal subgroup of $\widehat{G}^{0}$. The facts $G \cap H=\mathrm{Id}_{4}$ and $\widehat{G}^{0}=G H$ therefore prove $\widehat{G}^{0}$ to be the semidirect product [5] of $G \simeq \mathbb{C}_{+}$and $H$. 
Consider, besides, the three subgroups of $H$ formed by matrices of the form $V_{\kappa}, W_{\beta}, X_{\gamma}$, respectively; they are all normal subgroups of $H$ and representations of $\mathbb{C}_{+}$, and their pairwise intersections are $\left\{\mathrm{Id}_{4}\right\}$. Therefore, writing $\times$ as the direct product and $\ltimes$ as the semidirect product, we have

$$
\widehat{G}^{0}=G \ltimes H \simeq \mathbb{C}_{+} \ltimes\left(\mathbb{C}_{+} \times \mathbb{C}_{+} \times \mathbb{C}_{+}\right) .
$$

So far we have assumed $S_{1}=S_{2}=S_{3}=\mathbb{C}$; were that false for any of them, say, $S_{i}$, it would still have to be the underlying set of a algebraic subgroup of the additive group $\mathbb{C}_{+}$, since each of $\kappa, \beta, \gamma$ comes from one quadrature; indeed, if we consider $L_{1}$ as our base group, we have $\operatorname{Gal}\left(L_{2} \mid L_{1}\right)=H$ and $\mu=0$ in formula (28), which in turn yields additive actions on $\int V$ :

$$
\int u \mapsto \int u-\kappa, \quad \int v_{1} \mapsto \int v_{1}+\gamma, \quad \int v_{2} \mapsto \int v_{2}+\beta .
$$

Parameters $\kappa, \beta, \gamma$ thus belong to an algebraic subgroup of $\mathbb{C}_{+}$(i.e., $\mathbb{C}$ or $\{0\}$ ), so

$$
S_{i} \in\{\{0\}, \mathbb{C}\}, \quad i=1,2,3
$$

(recall Remark 2.2(2)). However, $\kappa$ is not identically zero. If it were, (30) would then prove $\int u$ invariant under any $\sigma \in \widehat{G}^{0}$; this, the logarithm in $\int u$ and Remark 5.3 are obviously in contradiction with the normality of $L_{2} \mid K . \gamma$ is not identically zero, either; otherwise, the product in $\widehat{G}^{0}$ would not be defined. Therefore

$$
S_{1}=S_{3}=\mathbb{C}, \quad S_{2} \in\{\{0\}, \mathbb{C}\} .
$$

Resetting $K_{1}$ as our base field in order to obtain the remaining parameter $\mu$, and using both the factorization $a_{\mu, \kappa, \beta, \gamma}=U_{\mu} V_{\kappa} W_{\beta} X_{\gamma}$ and the isomorphism provided by the second part of Fundamental Theorem 2.5,

$$
\operatorname{Gal}\left(L_{1} \mid K_{1}\right) \simeq \operatorname{Gal}\left(L_{2} \mid K_{1}\right) / \operatorname{Gal}\left(L_{2} \mid L_{1}\right),
$$

we actually have (in this case) a splitting of $\operatorname{Gal}\left(L_{2} \mid K_{1}\right)$ as the semidirect product

$$
\widehat{G}^{0}=G \ltimes H=G \ltimes\left(\widehat{G}^{0} / G\right) \simeq \mathbb{C}_{+} \ltimes\left(\mathbb{C}_{+} \times \mathbb{C}_{+} \times S_{2}\right) .
$$

Both this and condition (31) force $\widehat{G}^{0}$ to be isomorphic to one of the following:

$$
\mathbb{C}_{+} \ltimes\left(\mathbb{C}_{+} \times \mathbb{C}_{+} \times \mathbb{C}_{+}\right) \text {or } \mathbb{C}_{+} \ltimes\left(\mathbb{C}_{+} \times \mathbb{C}_{+}\right),
$$

non-commutative, in any case.

Remarks 6.1. Regarding the proof of Theorem 3.3:

1. In Step 3 the form of (24) clearly embodies our need for the whole fundamental matrix $\Psi$; in other words, solving the NVE is not enough to prove Theorem 3.3. Indeed, the theorems due to Ziglin and Morales-Ramis are of no use up to this step, since $\tilde{G}$ is a commutative group of unipotent (and thus resonant) matrices.

2. An alternative approach to Step 4. Recall the unipotent radical of any given linear algebraic group $G$ as being the (unique) largest closed, connected, normal subgroup formed by unipotent matrices in $G$. We know, thanks to [2, p. 27], that the unipotent radical of 
the symplectic group $\operatorname{Sp}(2, \mathbb{C})$ may be expressed, in an suitable basis $\left\{e_{1}, e_{2}, e_{3}, e_{4}\right\}$, as follows:

$$
G_{u}=\left\{\left(\begin{array}{cccr}
1 & \mu & \kappa+\mu \beta & \gamma \\
0 & 1 & \beta & \kappa \\
0 & 0 & 1 & -\mu \\
0 & 0 & 0 & 1
\end{array}\right): \mu, \kappa, \beta, \gamma \in \mathbb{C}\right\},
$$

the coordinates still being canonical. Using $\tilde{e}_{3}=-e_{3}$ we transform the fundamental matrix $\left(e_{1}, e_{2}, e_{3}, e_{4}\right)$ into $\left(e_{1}, e_{2}, \tilde{e}_{3}, e_{4}\right)$. The fact these are not canonical coordinates will not affect our result: the symplectic manifold and bundle and the Galois group will remain invariant.

Subsequent changes $\beta \mapsto-\beta, \gamma \mapsto \gamma+\mu \kappa$, in this order, make the representation turn into

$$
G_{u} \cong\left\{\left(\begin{array}{cc}
A & A B \\
0 & A
\end{array}\right): A=\left(\begin{array}{cc}
1 & \mu \\
0 & 1
\end{array}\right), B=\left(\begin{array}{cc}
-\kappa & \gamma \\
\beta & \kappa
\end{array}\right), \mu, \kappa, \beta, \gamma \in \mathbb{C}\right\},
$$

that is, exactly in the form (29) with $S_{i}=\mathbb{C}, i=1,2,3$.

Let us return to $\widehat{G}^{0}$. The fact that this group is a connected, normal and unipotent subgroup of the symplectic group assures $\widehat{G}^{0} \subset G_{u}$. This is just what was proven in Step 4.

3. An alternative ending to Step 5. In the general expression of $\widehat{G}^{0}$, as we know, the domain of definition for $\mu, \kappa, \gamma$ is all of $\mathbb{C}$, and the one for $\beta$ is either $\mathbb{C}$ once again or $\{0\}$; given any $a_{i}:=a_{\mu_{i}, \kappa_{i}, \beta_{i}, \gamma_{i}} \in \widehat{G}^{0}, i=1,2$, their commutator is

$$
\begin{aligned}
a_{1} a_{2} a_{1}^{-1} & a_{2}^{-1}=a_{\mu_{1}, \kappa_{1}, \beta_{1}, \gamma_{1}} a_{\mu_{2}, \kappa_{2}, \beta_{2}, \gamma_{2}} a_{\mu_{1}, \kappa_{1}, \beta_{1}, \gamma_{1}}^{-1} a_{\mu_{2}, \kappa_{2}, \beta_{2}, \gamma_{2}}^{-1} \\
\quad= & \left(\begin{array}{ccccc}
1 & 0 & \mu_{1} \beta_{2}-\beta_{1} \mu_{2} & 2\left(\mu_{1} \kappa_{2}-\mu_{2} \kappa_{1}\right)-\mu_{1} \beta_{2}\left(\mu_{1}+2 \mu_{2}\right)+\beta_{1} \mu_{2}\left(\mu_{2}+2 \mu_{1}\right) \\
0 & 1 & 0 & \beta_{1} \mu_{2}-\mu_{1} \beta_{2} \\
0 & 0 & 1 & 0 \\
0 & 0 & 0 & 1
\end{array}\right) .
\end{aligned}
$$

It is now a simple exercise to verify this is not identically equal to $\mathrm{Id}_{4}$, which also proves $\widehat{G}^{0}$ non-commutative.

4. Some comments on preliminary methodology and checks. Reliance on numerics not only provided significant preliminary information prior to the actual proof; it also shed some light into the ensuing algebraic framework, namely in the relationship between the monodromy group and the presumably larger one $\widehat{G}^{0}=\operatorname{Gal}\left(L_{2} \mid K_{1}\right)$ containing it. We first considered system (8), along with the related variational equations as given in (11), from a numerical point of view. Clearly, for $h \in\left(0, h^{*}\right)(8)$ has both a real and a purely imaginary period (with $\phi^{\prime}$ real in both cases). It is enough to take $\left(\phi(0), \phi^{\prime}(0)\right)=(0, \sqrt{2 h})$ as initial conditions and then real or imaginary times, respectively.

Let $M_{1}$ and $M_{2}$ be the monodromy matrices along the real and the imaginary periods, respectively. These matrices have the common structure

$$
M=\left(\begin{array}{rrrr}
1 & p & q & 0 \\
0 & 1 & 0 & -q \\
0 & 0 & 1 & p \\
0 & 0 & 0 & 1
\end{array}\right)
$$




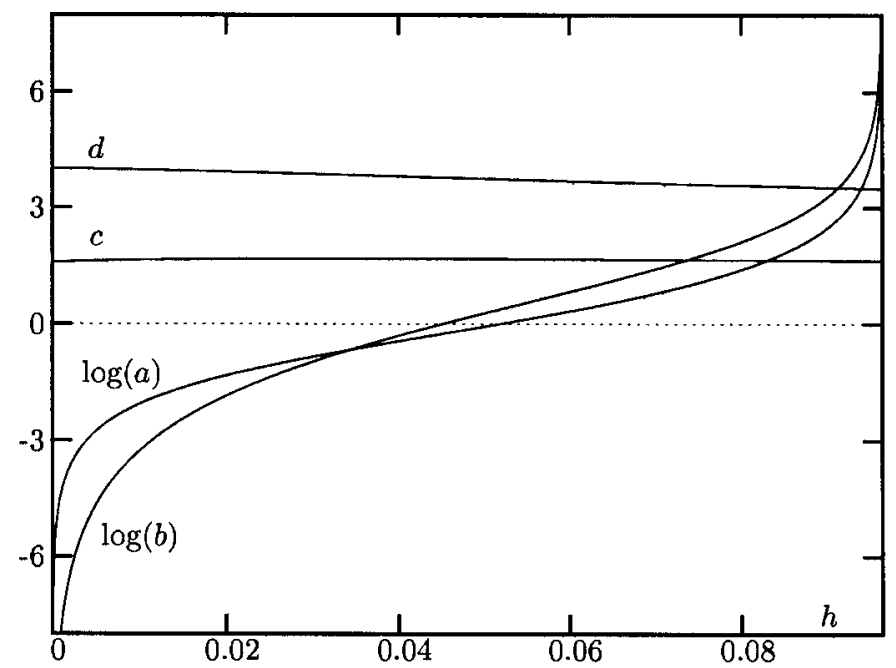

FIGURE 1. Values of parameters $a, b, c, d$ (first two in logarithmic scale) appearing in the monodromy matrices for real and purely imaginary period. See the text for details.

which, of course, turns out to be a particular case of (29). In the real period case $p=a i$, $q=b(1-i)$ has been found, and in the imaginary period case $p=c, q=d(1+i)$, with $a, b, c, d \in \mathbb{R}$, all of them positive. The computed values of $a, b, c, d$ are displayed, as a function of $h$, in Figure 1. Let $p_{1}, q_{1}$ and $p_{2}, q_{2}$ the $p, q$ entries in $M_{1}$ and $M_{2}$. These matrices commute if and only if $\Delta:=p_{1} q_{2}-q_{1} p_{2}=(i-1)(a d+b c)=0$. From the positive character of $a, b, c, d$ it follows $\Delta \neq 0$ for all $h \in\left(0, h^{*}\right)$. Furthermore, the coefficient of $(i-1)$ in $\Delta$ is far away from zero, except for small $h$, a domain amenable to perturbative computations.

The group generated by $M_{1}$ and $M_{2}$, which is a subgroup of the monodromy one and, hence, a subgroup of the Galois group, has the same structure as in (29) with $\beta=0$. This is in favour of the second of the options presented for $\widehat{G}^{0}$, i.e., $\widehat{G}^{0} \simeq \mathbb{C}_{+} \ltimes\left(\mathbb{C}_{+} \times \mathbb{C}_{+}\right)$.

\section{Concluding statements}

In [7], the authors start from (5) expressed in polar canonical coordinates and with scalings leading to the Hamiltonian

$$
\tilde{H}_{b, \omega}=H_{0}+\omega^{2} H_{2},
$$

where $\omega^{2}$ is assumed small enough, $H_{0}$ is the Hamiltonian of Kepler's classical problem in a reference frame rotating with angular velocity $b$, and $\tilde{H}_{\omega, \omega}$ is Hill's Hamiltonian. The strategy followed henceforth is based in proving there is no first integral $\Phi$ at a time independent of $\tilde{H}_{b, \omega}$ and analytical with respect to $\omega$ in an open neighbourhood of $\omega=0$.

The authors presumably afford their non-integrability proof restricting it to first integrals which are analytical with respect to the conjugate variables and the parameter $\omega$; in other words, their proof does not deny, for instance, the existence of additional first 
integrals meromorphic with respect to phase variables and satisfying the Liouville-Arnold hypotheses. That denial, which discards any restriction concerning $\omega$, comes precisely from our proof.

As for [11], the proof given there is of algebraic non-integrability; using his own generalization of a method nearly 100 years old, the author establishes there is no second integral of motion for the HP which is polynomial with respect to phase variables at a given (arbitrary) level of energy. Spurious parameters such as momentum are not considered here, but the constraint of algebraic dependence is still far stronger than our hypothesis of meromorphic dependence on canonical variables.

Acknowledgements. The research of J.J.M.-R. and C.S. has been partially supported by grant CIRIT 2001 SGR-70 (Catalonia). Furthermore the first and second authors have been supported by grants BFM2003-09504-C02-02 and BFM2003-09504-C02-01 (MCYT, Spain), respectively.

\section{REFERENCES}

[1] V. I. Arnold. Mathematical Methods of Classical Mechanics. Springer, New York, 1980.

[2] R. C. Churchill, D. L. Rod and M. F. Singer. Group-theoretic obstructions to integrability. Ergod. Th. \& Dynam. Sys. 15 (1995), 15-48.

[3] P. Du Val. Elliptic Functions and Elliptic Curves. Cambridge University Press, Cambridge, 1973.

[4] G. W. Hill. Researches in the Lunar theory. Amer. J. Math. 1 (1878), 5-6, 129-147, 245-260.

[5] J. Humphreys. Linear Algebraic Groups. Springer, New York, 1981.

[6] E. L. Ince. Ordinary Differential Equations. Dover, New York, 1956.

[7] E. Meletlidou, S. Ichtiaroglou and F. J. Winterberg. Non-integrability of Hill's Lunar problem. Celestial Dynamics and Dynamical Astronomy 80 (2001), 145-156.

[8] J. J. Morales-Ruiz. Differential Galois Theory and Non-Integrability of Hamiltonian Systems. Birkhäuser, Basel, 1999.

[9] J. J.Morales-Ruiz and J. P. Ramis. Galoisian obstructions to integrability of Hamiltonian systems, I. Methods Appl. Anal. 8 (2001), 33-96.

[10] J. J. Morales-Ruiz, J. P. Ramis and C. Simó. Integrability of Hamiltonian systems and differential Galois groups of higher variational equations, to appear.

[11] S. T. Sadetov. On algebraic integrals of Hill (sic) problem and restricted circular planar three-body problem on a level of energy. Regul. Chaotic Dyn. to appear.

[12] C. Simó. An overview on some problems in Celestial Mechanics, included in 'Iniciación a los sistemas dinámicos', Cursos de Verano, Universidad Complutense de Madrid, El Escorial, 1997. Available at http://www-ma1.upc.es/escorial/index.html.

[13] C. Simó and T. J. Stuchi. Central stable/unstable manifolds and the destruction of KAM tori in the planar Hill problem. Physica D 140(1-2) (2000), 1-32.

[14] M. F. Singer and M. van der Put. Galois Theory of Linear Differential Equations (Grundlehren der mathematischen Wissenschaften, 328). Springer, Berlin, 2003.

[15] E. L. Stiefel and G. Scheifele. Linear and Regular Celestial Mechanics. Springer-Verlag, Berlin and Heidelberg, 1971.

[16] V. G. Szebehely. Theory of Orbits. Academic Press, New York, 1967.

[17] E. T. Whittaker and G. N. Watson. A Course of Modern Analysis. Cambridge University Press, Cambridge, 1940.

[18] S. L. Ziglin. Branching of solutions and nonexistence of first integrals in Hamiltonian mechanics. I. Funct. Anal. Appl. 16 (1982), 181-189. 